\title{
A Feynman-Kac Path-Integral Implementation for Poisson's Equation
}

\author{
Chi-Ok Hwang and Michael Mascagni \\ Department of Computer Science, Florida State University, 203 Love Building \\ Tallahassee, FL 32306-4530
}

\begin{abstract}
This study presents a Feynman-Kac path-integral implementation for solving the Dirichlet problem for Poisson's equation. The algorithm is a modified "walk on spheres" (WOS) that includes the FeynmanKac path-integral contribution for the source term. In our approach, we use the Poisson kernel instead of simulating Brownian trajectories in detail to implement the path-integral computation. We derive this approach and provide results from a numerical experiment on a two-dimensional problem as verification of the method.
\end{abstract}

\section{Introduction}

Since Müller proposed the "walk on spheres" (WOS) method for solving the Dirichlet boundary value problems for the Laplace equation [1], WOS has been a popular method. In addition, this random-walk based approach has been extended to solve other, more complicated, partial differential equations including Poisson's equation, and the linearized Poisson-Boltzmann equation [2-8]. In WOS, instead of using detailed Brownian trajectories inside the domain, discrete jumps are made using the uniform first-passage probability distribution of the sphere. In this paper, this WOS method is combined with the Feynman-Kac formulation to solve the Dirichlet boundary value problem for Poisson's equation. Even though the Feynman-Kac method is well known among mathematicians and mathematical physicists, [9-11], as a computational technique, it has not been much implemented, even for simple cases, despite the fact that some modified WOS methods are mathematically derivable from the Feynman-Kac formulation [4]. We thus feel that it is worthwhile to implement the Feynman-Kac formulation for some simple problems to show its utility. Here, we implement the Feynman-Kac formulation for a simple Poisson problem. Instead of simulating the detailed Brownian trajectory, we use discrete WOS jumps together with the Poisson kernel to incorporate the source term in the Poisson equation. In previous work of others, different Green's functions have been used $[2,4,8]$ for the source term in the Poisson's equation, but the Poisson kernel has never been used. Here, we interpret the Poisson kernel as the probability density distribution of a Brownian trajectory inside a ball during its passage from the ball's center to an exit point on the ball's boundary. This is used in place of a direct simulation of the Brownian trajectory because the Poisson kernel gives the Brownian walker's density distribution inside the ball.

V.N. Alexandrov et al. (Eds.): ICCS 2001, LNCS 2073, pp. 1282-1288 2001.

(C) Springer-Verlag Berlin Heidelberg 2001 
This paper is organized as follows. In Section 2, we explain how to implement the Feynman-Kac path-integral method for the Dirichlet problem for Poisson's equation. In Section 3, we give a numerical example. In Section 4, conclusions are presented and future work is discussed.

\section{Modified "walk on spheres"}

In this section, we explain how to combine the WOS method [1] with the Feynman-Kac path-integral representation for solving the Dirichlet problem for Poisson's equation. Our implementation is based on the well-known FeynmanKac representation of the solution to the Dirichlet problem for Poisson's equation. Recall that the Dirichlet problem for the Poisson's equation is:

$$
\begin{gathered}
\frac{1}{2} \Delta u(\mathbf{x})=-q(\mathbf{x}), \quad \mathbf{x} \in \Omega \\
u(\mathbf{x})=f(\mathbf{x}), \quad \mathbf{x} \in \partial \Omega
\end{gathered}
$$

The solution to this problem, given in the form of the path-integral with respect to standard Brownian motion $X_{t}^{\mathbf{x}}$, is as follows $[9,10]$ :

$$
u(\mathbf{x})=E\left[\int_{0}^{\tau_{D}^{\mathbf{x}}} q\left(X_{t}^{\mathbf{x}}\right) d t\right]+E\left[f\left(X_{\tau_{D}^{\mathbf{x}}}^{\mathbf{x}}\right)\right],
$$

where $\tau_{D}^{\mathbf{x}}=\left\{t: X_{t}^{\mathbf{x}} \in \partial \Omega\right\}$ is the first passage time and $X_{\tau_{D}^{\mathbf{x}}}^{\mathbf{x}}$ is the first passage location on the boundary, $\partial \Omega^{1}$.

Instead of simulating the detailed irregular motion of the Brownian trajectories, we use the Poisson kernel for a ball [10] with WOS as a probability density function:

$$
K(\mathbf{x}, \mathbf{z})=\frac{\Gamma(d / 2)}{2 \pi^{d / 2} r} \frac{r^{2}-|\mathbf{x}-\mathbf{a}|^{2}}{|\mathbf{x}-\mathbf{z}|^{d}}, \quad \mathbf{x} \in B(\mathbf{a}, r), \quad \mathbf{z} \in S(\mathbf{a}, r) .
$$

Here, $B(\mathbf{a}, r)$ is a ball with center $\mathbf{a}$ and radius $r, \mathbf{z}$ is the first passage location on the surface of the ball, $S(\mathbf{a}, r)$, and $d$ is dimension. We interpret the Poisson kernel as the probability density distribution of the Brownian trajectory inside the ball during its passage from the center to the boundary.

We construct a Brownian trajectory as a sequence of discrete jumps from ball centers to ball surfaces. Using the Poisson kernel, the first term of Eq. 3 for each $i$ th ball of a WOS (Brownian) trajectory becomes

$$
E\left[E\left\{\tau_{D}^{\mathbf{x}_{\mathbf{i}}}\right\} \int_{B_{i}} q(\mathbf{x}) K(\mathbf{x}, \mathbf{z}) d \mathbf{x}\right] .
$$

\footnotetext{
${ }^{1}$ Here, we assume that $E\left[\tau_{D}^{\mathbf{x}}\right]<\infty$ for all $\mathbf{x} \in \Omega, f(\mathbf{x})$ and $q(\mathbf{x})$ are continuous and bounded, and that the boundary, $\partial \Omega$, is sufficiently smooth so as to ensure the existence of a unique solution, $u(\mathbf{x})$, that has bounded, continuous, first-order and second-order partial derivatives in any interior subdomain $[9,10]$.
} 


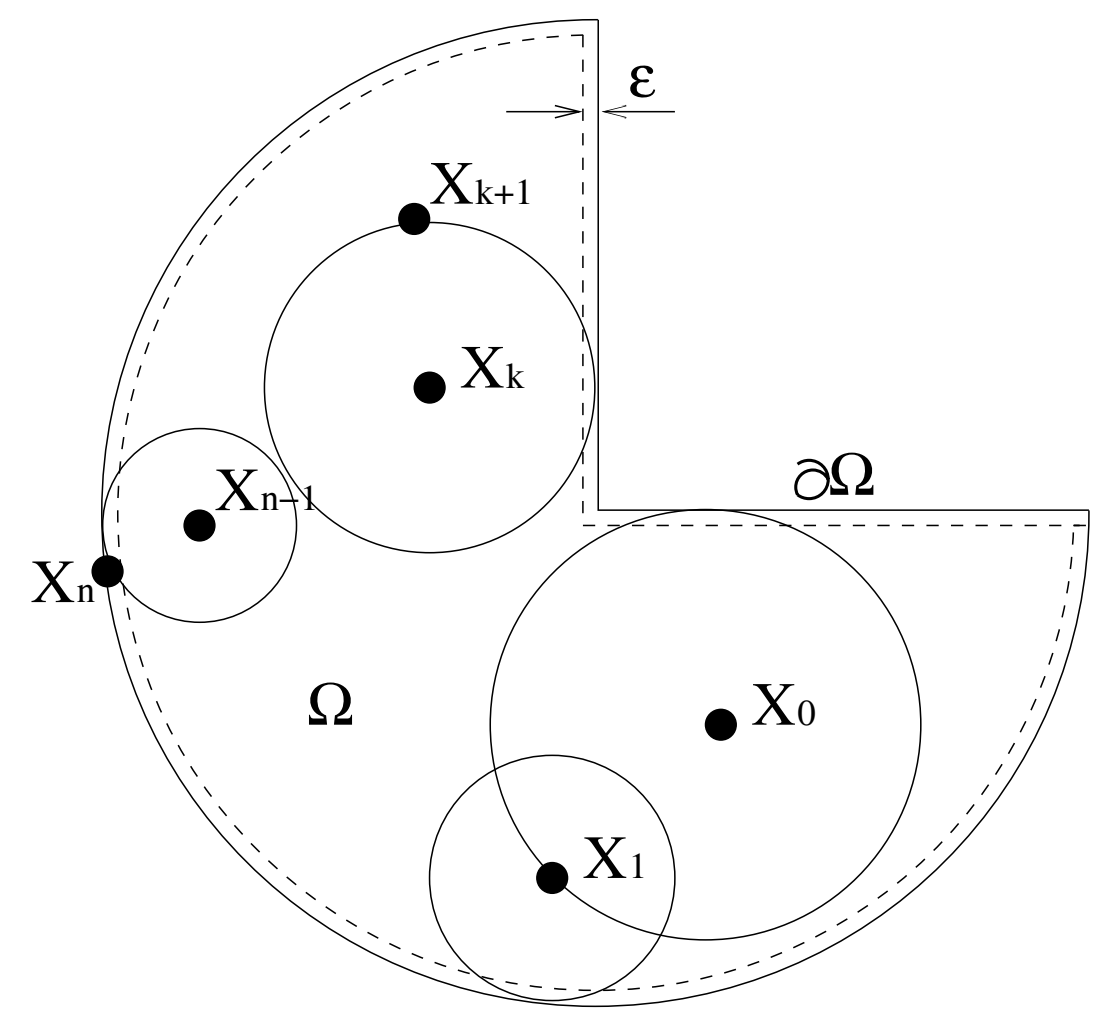

Fig. 1. Modified WOS. $X_{0}, X_{1}, \ldots, X_{k}, \ldots, X_{n}$ are a series of discrete jumps of a Brownian trajectory which terminates on absorption in the $\epsilon$-absorption layer.

Here, $B_{i}$ is the volume of the $i$ th ball and so the proceeding is a volume integral. It is also well known that the mean first passage time in this situation is $E\left[\tau_{D}^{\mathbf{x}_{D}^{i}}\right]=$ $r_{i}^{2} / 2 d$ in $d$ dimensions $[12,13]$. Notice that the integral is improper at $\mathbf{z}$ when $\mathbf{x}=\mathbf{z}$, the exit point.

Eq. 5 readily permits the use of WOS to eliminate the need to compute the detailed Brownian trajectory. Instead, a series of discrete jumps in continuous space terminating on the boundary, $\partial \Omega$, is used. Jumping from ball to ball never permits a trajectory to land exactly on the boundary. Thus we use the standard WOS approach of "fattening" the boundary by $\epsilon$ to create a capture region that is used to terminate the walk [2]. The error associated with this approximation has been theoretically estimated in previous WOS methods [6, 8].

We wish to compute the solution to the Dirichlet problem for Poisson's equation at $\mathbf{x}_{\mathbf{0}}$. For each Brownian trajectory starting at $\mathbf{x}_{\mathbf{0}}$, with an $\epsilon$-absorption layer, we accumulate the internal contribution for each ball and the functional value of the boundary condition at the final exit location on $\partial \Omega$. And so, an estimate for the solution at $\mathbf{x}_{\mathbf{0}}$ is given by the statistic 
Table 1.

\begin{tabular}{|c|c|c|c|c|c|}
\hline $\mathrm{r}$ & $\theta$ & Exact & Monte Carlo & variance & Average number of steps \\
\hline 0.1244 & -0.7906 & 0.8623 & 0.8676 & 0.0971 & 13.04 \\
0.2320 & -0.0274 & 0.9678 & 0.9694 & 0.0039 & 8.70 \\
0.2187 & -3.3975 & 0.4308 & 0.4348 & 0.1826 & 13.54 \\
0.1476 & -4.1617 & 0.4695 & 0.4564 & 0.0886 & 13.01 \\
0.0129 & -1.4790 & 0.8890 & 0.8843 & 0.0654 & 12.24 \\
\hline
\end{tabular}

$$
S_{N}=\frac{1}{N} \sum_{i=1}^{N} Z_{i}
$$

where $N$ is the number of trajectories and each statistic, $Z_{i}$ is given by

$$
Z_{i}=\sum_{i=1}^{n_{i}}\left[E\left\{\tau_{D}^{\mathbf{x}_{\mathbf{i}}}\right\} \int_{B\left(\mathbf{x}_{\mathbf{i}}, r_{i}\right)} q(\mathbf{x}) K(\mathbf{x}, \mathbf{z}) d \mathbf{x}+f\left(X_{\tau_{D}^{\mathbf{x}}}^{\mathbf{x}}\right)\right] .
$$

Here, $n_{i}$ is the number of WOS steps needed for the $i$ th Brownian trajectory to terminate in the $\epsilon$-absorption layer.

\section{$3 \quad$ Numerical Experiments}

In this section, we demonstrate our Feynman-Kac implementation by solving numerically a boundary value problem for Poisson's equation. We use as our domain, $\Omega$, the unit disk minus the first quadrant, which was used in previous research by DeLaurentis and Romero [8] (See Fig 1.):

$$
\Omega=\{(r, \theta): 0<r<1,-3 \pi / 2<\theta<0\} .
$$

We consider the Poisson equation:

$$
\frac{1}{2} \Delta u(\mathbf{x})=-\left(1-\frac{r^{2}}{2}\right) e^{-r^{2} / 2}
$$

with the boundary conditions $u(r, 0)=e\left(-r^{2} / 2\right), u(r,-3 \pi / 2)=-r^{1 / 3}+e\left(-r^{2} / 2\right)$, and $u(1, \theta)=\sin (\theta / 3)+e^{-1 / 2}$. The known analytic solution is

$$
u(r, \theta)=r^{1 / 3} \sin (\theta / 3)+e^{-r^{2} / 2} .
$$

For this two-dimensional problem, the Poisson kernel is

$$
K(\mathbf{x}, \mathbf{z})=\frac{1}{2 \pi r} \frac{r^{2}-|\mathbf{x}-\mathbf{a}|^{2}}{|\mathbf{x}-\mathbf{z}|^{2}}, \quad \mathbf{x} \in B(\mathbf{a}, r), \quad \mathbf{z} \in S(\mathbf{a}, r),
$$

and one random estimate is

$$
Z_{i}=\sum_{i=1}^{n} \frac{r_{i}^{2}}{4} \int_{B\left(\mathbf{x}_{\mathbf{i}}, r_{i}\right)} q(\mathbf{x}) K(\mathbf{x}, \mathbf{z}) d \mathbf{x}+f\left(X_{\tau_{D}^{\mathbf{x}}}^{\mathbf{x}}\right) .
$$




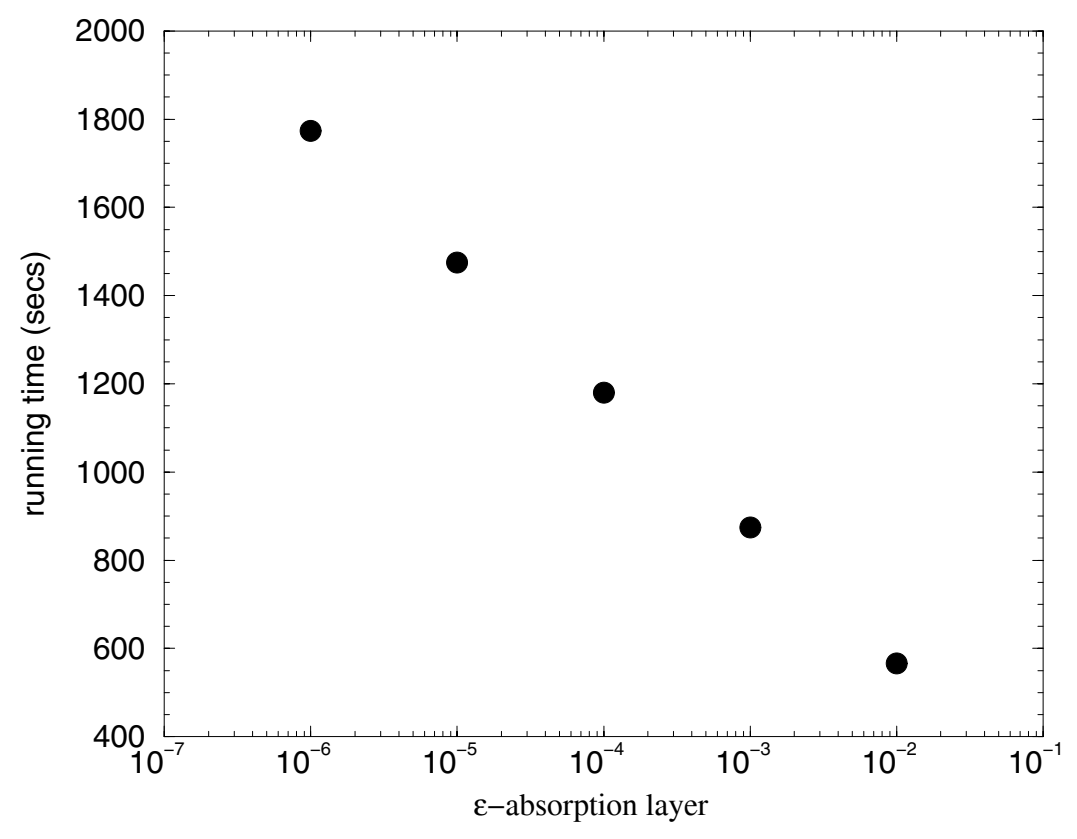

Fig. 2. Running time v.s. the thickness of the $\epsilon$-absorption layer. This shows the usual relation for WOS, running time (proportional to number of WOS steps for each Brownian trajectory) on the order of $|\log \epsilon|$.

Table I shows our simulation results for the solution at five different points. The absorption layer thickness is $\epsilon=10^{-4}$, and the number of trajectories for each run is $N=10^{3}$.

The errors associated with this implementation are (1) the error associated with the number of trajectories (sampling error), (2) the error associated with the $\epsilon$-absorption layer, and (3) the error associated with the integration used for the source term. We can reduce the statistical sampling error by increasing the number of trajectories. The error associated with the $\epsilon$-absorption layer can be reduced by reducing $\epsilon$, the $\epsilon$-absorption layer thickness. However, increasing the number of trajectories will increase the running time linearly, while reducing $\epsilon$ will increase running time on the order of $|\log \epsilon|$. In Fig. 2, we see the usual relationship between running time and the thickness of the $\epsilon$-absorption layer in WOS: on the order of $|\log \epsilon|$. The reason for this is that the running time increases proportionally to the number of WOS steps, $n_{i}$, as an integration is required for the source term in each WOS step. The error from the $\epsilon$-absorption layer can be investigated empirically if we have enough trajectories so that the statistical sampling error is much smaller than the error from the $\epsilon$-absorption layer. Fig. 3 shows the empirical results with $10^{6}$ Brownian trajectories: the $\epsilon$-layer error grows linearly in $\epsilon$ for small $\epsilon$. 


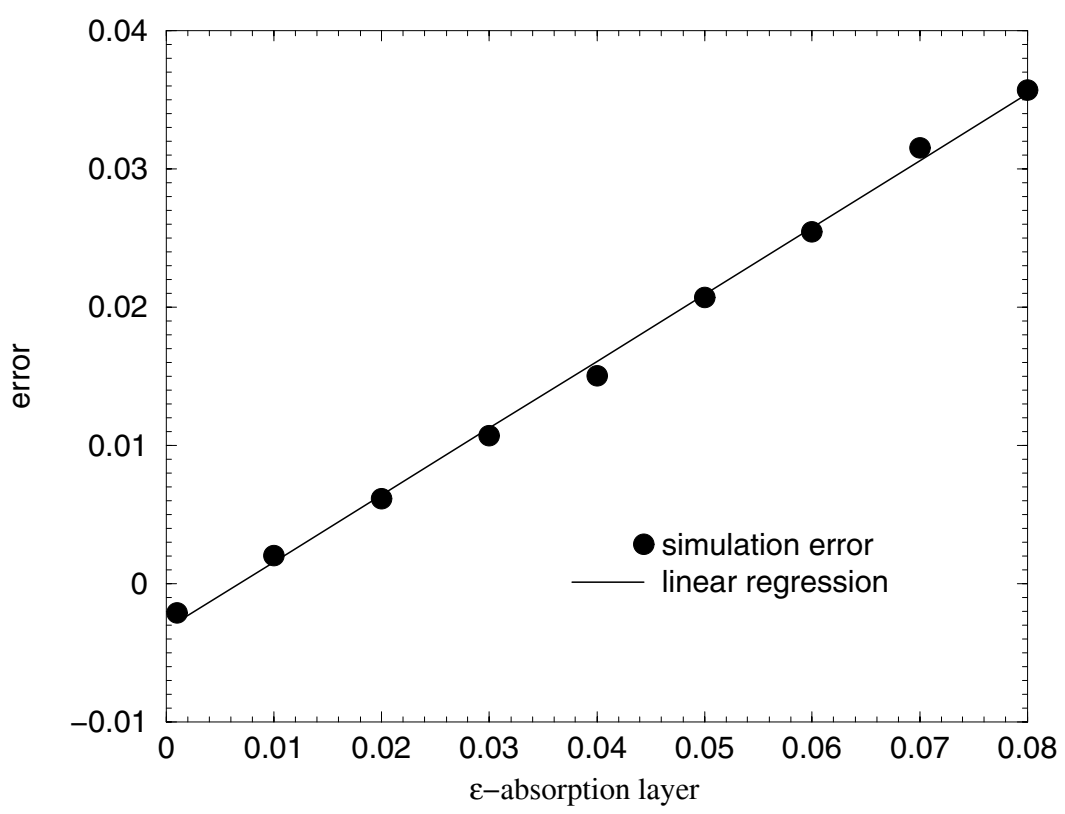

Fig. 3. Error arising from the $\epsilon$-absorption layer with $10^{6}$ Brownian trajectories. The error is linear in $\epsilon$.

\section{Conclusions and Future Work}

In this study, we implemented the Feynman-Kac path-integral representation of the solution to the Dirichlet problem for Poisson's equation combining the well known WOS method with use of the Poisson kernel as a probability density function. Using the Poisson kernel inside each WOS step, we avoid the need for detailed information about the Brownian trajectory inside the spherical domain. The Brownian trajectory is thus constructed as a series of discrete jumps using WOS with the source contribution inside each WOS step computed using the Poisson kernel.

Recently, we developed a modified WOS algorithm for solving the linearized Poisson-Boltzmann equation (LPBE) [7] in a domain $\Omega$ :

$$
\begin{gathered}
\Delta \psi(\mathbf{x})=\kappa^{2} \psi(\mathbf{x}), \quad \mathbf{x} \in \Omega, \\
\psi(\mathbf{x})=\psi_{0}(\mathbf{x}), \quad \mathbf{x} \in \partial \Omega .
\end{gathered}
$$

Here, $\kappa$ is called the inverse Debye length [14]. We used a survival probability, which was obtained by reinterpreting a weight function in a previously modified WOS method [4]. This survival probability enabled us to terminate some Brownian trajectories during WOS steps. This method can be combined with the method described in this paper to solve the Dirichlet boundary value problem for $\Delta \psi(\mathbf{x})-\kappa^{2} \psi(\mathbf{x})=-g(\mathbf{x})$. This will be the subject of a future study. 


\section{References}

1. M. E. Müller. Some continuous Monte Carlo methods for the Dirichlet problem. Ann. Math. Stat., 27:569-589, 1956.

2. K. K. Sabelfeld. Monte Carlo Methods in Boundary Value Problems. SpringerVerlag, Berlin, 1991.

3. A. Haji-Sheikh and E. M. Sparrow. The solution of heat conduction problems by probability methods. Journal of Heat Transfer, 89:121-131, 1967.

4. B. S. Elepov and G. A. Mihailov. The "Walk On Spheres" algorithm for the equation $\Delta u-c u=-g$. Soviet Math. Dokl., 14:1276-1280, 1973.

5. T. E. Booth. Exact Monte Carlo solution of elliptic partial differential equations. J. Comput. Phys., 39:396-404, 1981.

6. T. E. Booth. Regional Monte Carlo solution of elliptic partial differential equations. J. Comput. Phys., 47:281-290, 1982.

7. C.-O. Hwang and M. Mascagni. Efficient modified "Walk On Spheres" algorithm for the linearized Poisson-Boltzmann equation. Appl. Phys. Lett., 78(6):787-789, 2001.

8. J. M. DeLaurentis and L. A. Romero. A Monte Carlo method for Poisson's equation. J. Comput. Phys., 90:123-139, 1990.

9. M. Freidlin. Functional Integration and Partial Differential Equations. Princeton University Press, Princeton, New Jersey, 1985.

10. K. L. Chung and Z. Zhao. From Brownian Motion to Schrödinger's Equation. Springer-Verlag, Berlin, 1995.

11. K. K. Sabelfeld. Integral and probabilistic representations for systems of elliptic equations. Mathematical and Computer Modelling, 23:111-129, 1996.

12. L. H. Zheng and Y. C. Chiew. Computer simulation of diffusion-controlled reactions in dispersions of spherical sinks. J. Chem. Phys., 90(1):322-327, 1989.

13. S. Torquato and I. C. Kim. Efficient simulation technique to compute effective properties of hetergeneous media. Appl. Phys. Lett., 55:1847-1849, 1989.

14. R. Ettelaie. Solutions of the linearized Poisson-Boltzmann equation through the use of random walk simulation method. J. Chem. Phys., 103(9):3657-3667, 1995. 